\title{
Study of hyperparathyroidism among patients with chronic kidney disease at sohag university hospital
}

\author{
Mahmoud Kh. Mahmoud (Resident ) ; Ahmed M. Aly(MD); Nayel A. Zaki(MD); \\ Ali T. Ali(MD)
}

\begin{abstract}
Objectives:The aim of this study is to asses prevalence \& clinical outcome of hyperparathyroidism among CKDpatients either on dialysis or not in Sohag University Hospital 1.To estimate serum intact parathormone(iPTH) and other biochemical parameters (calcium, inorganic phosphate, urea and creatinine)in CKDpatients2. To compare and find out correlation between serum intact parathormone and biochemicalparameters in the study group.

Patients \&Methods: For the study of 5 stages of Chronic Kidney Disease(CKD) totally 100 patients in different stages inthe age group of 20-60 years were taken. Serum parathormone and other biochemical parameters were estimated using standard methods.Pelvi-abdominal ultrasonography,Plain x-ray,ECG \& Echocardiography were also done.
\end{abstract}

Results:66 patients (66.00\%) with PTH values above the normal range (hyperparathyroidism) $(>72 \mathrm{pg} / \mathrm{ml})$, with mean PTH $=481.92 \pm 608.05$, and this is the prevalence rate of secondary hyperparathyroidism in the study population. The serum PTH levels were higher even in early stages of CKD and the higher values are directly related to the stage of CKD. In the study, Serum phosphorus was found to be significantly positively correlated to PTH both in the total study population and in patients with hyperparathyroidism.The PTH was found to be significantly negatively correlated with serum total and ionized calcium of the patients, both in the total study population and in patients with hyperparathyroidism. Also the findings of this study as regard echocardiography in CKD patients;Left ventricular dysfunction was the commonest cardiovascular abnormality. LVH was the most common echocardiographic abnormality in CKD cases. Diastolic function was deranged in more number of patients as compared to systolic function in patients with CKD.

Conclusion: The estimation of serum PTH and other biochemical parameters can help to diagnose the secondary hyperparathyroidism in the early stage of CKD which inturn has advantage to manage the patients of CKD accordingly to prevent the future complications.

\section{Introduction}

Today CKD is a major health concern affecting $5-10 \%$ of the population globally ${ }^{1}$. The global incidence and prevalence of CKD is growing, mainly attributed to the ageing population and the concomitant increase in CKD risk factors, such as hypertension and diabetes. The age-adjusted mortality risk is increased already in early stages of CKD and increases further as the deterioration in renal function

progresses $^{2}$. In patients with CKD stage 5 , also called end stage renal disease (ESRD), the five-year survival rate is approximately $50 \%{ }^{3}$.

Secondary hyperparathyroidism is a common complication of chronic kidney disease (CKD), and is characterized by elevated levels of serum parathyroid hormone (PTH) and abnormalities in bone and mineral metabolism ${ }^{4}$. 
This serious disorder arises from disturbances in the regulation of intracellular and extracellular levels of PTH, calcium, phosphorus, and vitamin D (calcitriol), which become more severe as kidney function declines 5 .

The mechanisms by which the decrease in plasma calcium and vitamin D (calcitriol) and the increase in plasma phosphate in advanced stages of chronic renal failure stimulate PTH secretion became more obvious during the last decade $^{6}$.

These disturbances, in addition to several long known indirect effects, they all have also been shown to act directly on the parathyroid cell, via a cell membrane receptor for calcium ${ }^{7}$, a nuclear receptor for calcitriol $^{8}$, and a yet unknown cellular target for phosphate 9

In chronic renal failure, secondary hyperparathyroidism is associated with significant clinical risks, including risks for renal bone disease, cardiovascular and death, as well as less frequently recognized neurotoxicities and endocrinopathies ${ }^{10}$.

The bone, as one of the main organs for handling calcium and phosphate, is dependent on a well-regulated mineral metabolism to maintain its normal function, and as a consequence bone abnormalities are frequently found in the CKD population. The alterations in bone morphology associated with CKD are termed renal osteodystrophy, and encompasses changes in bone turnover, mineralization and bone volume. Renal osteodystrophy may result in fractures, bone pain and impaired linear growth inchildren ${ }^{11}$.

In parallel with bone abnormalities there is an increase in extraosseous calcifications in CKD, most importantly in the vasculature. Vascular calcification develops as a result of an imbalance between inducers and inhibitors of the calcification process, and several known inhibitors, including Fetuin-A and matrix Gla-protein, are reduced in CKD patients $^{12}$. Accordingly, vascular calcification is a major concern in CKD patients with increased prevalence already at CKD stage 3 (40\% compared to $13 \%$ in healthy individuals), and is a universal phenomenon in ESRD (approximately 80\%). Vascular calcification is closely related to and considered to be a valid surrogate for the longitudinal risk of CVD and mortality ${ }^{13}$. Although the molecular mechanism behind vascular calcification is not fully elucidated it appears to be a combination of passive deposition and an active cellular process. The passive deposition is caused by precipitation of high circulating concentrations of calcium and phosphate into the vascular wall, while the active process is commonly viewed as a dedifferentiation of vascular cells into bone-like cells, triggered by various stimuli ${ }^{14}$.

\section{Patients and Methods}

The aim of this study is to asses prevalence \& clinical outcome of hyperparathyroidism among CKDpatients either on dialysis or not in Sohag University Hospital.

This study was done in the nephrology clinic and the hemodialysis unit at Sohag University Hospital.

It was conducted on 100 patients with $\mathrm{CKD}$, either on regular hemodialysis or not.

The following patients will be excluded from the study:

- Patients with primary parathyroid gland disease or malignancy.

- Patients with known bone disease or malignancy.

- Postmenopausal women.

- Elderly patients above 60 years old. 
SOHAG MEDICAL JOURNAL Study of hyperparathyroidism among patients with chronic

- Patients on phenytoin, steroids or phenobarbitone as they affect bone biochemical factors.

All patients involved in the study were subjected to the following:

\section{A) Careful medical history:}

- Personal history.

- Present history including the original kidney disease, time on dialysis (in months), any cardiovascular, chest disease, diabetic status...

B) Full clinical examination:

- Careful general examination including heart, chest, abdominal and neurological examination

C) Laboratory investigations: Which included:

- Urine analysis.

- Blood urea and serum creatinine level ( will be done before and after dialysis in patients on hemodialysis)

\section{Results}

Our study included 100 patients with CKD either on dialysis or not randomly selected from Dialysis Unit and Nephrology Clinic in Sohag University Hospital.

Table 1 shows that, the mean age of the patients was $44.13 \pm 9.78$ years (ranged from 18 59 years), 63 males $(63 \%)$ and 37 females $(37 \%)$ were included, and they were on different stages of CKD each stage from stage 1 to 4 included $10(10.00 \%)$ patients and stage 5 included $60(60.00 \%)$ patients who were on haemodialysis treatment for duration for $6.58 \pm 3.09$ years. 31 patients $(31.00 \%)$ were diabetic who were on oral hypoglycemic treatment 13 patients and on insulin therapy 18 patients and 33 patients $(33.00 \%)$ have history of hypertension .3 patients $(3.00 \%)$ were previously diagnosed to have IHD. 
SOHAG MEDICAL JOURNAL Study of hyperparathyroidism among patients with chronic

Table.1 Demographic data of study populations

\begin{tabular}{|c|c|}
\hline Variable & Summary statistics \\
\hline $\begin{array}{l}\text { Age/year } \\
\text { Mean } \pm \text { SD } \\
\text { Median (range) }\end{array}$ & $\begin{array}{c}44.13 \pm 9.78 \\
45(18-59)\end{array}$ \\
\hline $\begin{array}{l}\text { Gender } \\
\text { Males } \\
\text { Females }\end{array}$ & $\begin{array}{l}63(63.00 \%) \\
37(37.00 \%)\end{array}$ \\
\hline $\begin{array}{l}\text { Hypertension } \\
\text { No } \\
\text { Yes } \\
\end{array}$ & $\begin{array}{l}67(67.00 \%) \\
33(33.00 \%)\end{array}$ \\
\hline $\begin{array}{l}\text { DM } \\
\text { No } \\
\text { Yes }\end{array}$ & $\begin{array}{c}69(69 \%) \\
31(31.00 \%)\end{array}$ \\
\hline $\begin{array}{l}\text { Treatment } \\
\text { No } \\
\text { Oral hypoglycemic } \\
\text { Insulin }\end{array}$ & $\begin{array}{l}69(69.00 \%) \\
13(13.00 \%) \\
18(18.00 \%)\end{array}$ \\
\hline $\begin{array}{l}\text { IHD } \\
\text { No } \\
\text { Yes }\end{array}$ & $\begin{array}{l}97(97 \%) \\
3(3.00 \%)\end{array}$ \\
\hline $\begin{array}{l}\text { Stages of CKD } \\
1 \\
2 \\
3 \\
4 \\
5 \\
\end{array}$ & $\begin{array}{l}10(10.00 \%) \\
10(10.00 \%) \\
10(10.00 \%) \\
10(10.00 \%) \\
60(60.00 \%) \\
\end{array}$ \\
\hline $\begin{array}{l}\text { Dialysis duration if stage }=\mathbf{5 D} \\
\text { Mean } \pm \text { SD } \\
\text { Median (range) }\end{array}$ & $\begin{array}{l}6.58 \pm 3.09 \\
5(2-16)\end{array}$ \\
\hline $\begin{array}{l}\text { Bone density } \\
\text { Normal } \\
\text { Osteopenia } \\
\text { Osteoporosis } \\
\text { Osteomalacia } \\
\end{array}$ & $\begin{array}{c}55(55.00 \%) \\
29(29.00 \%) \\
13(13.00 \%) \\
3(3.00 \%)\end{array}$ \\
\hline
\end{tabular}

By evaluation of serum levels of the following biochemical parameters; the mean serum Ca level of the patients was $8.69 \pm 1.30 \mathrm{mg} / \mathrm{dl}$, serum ionized Ca level of the patients was $1.04 \pm 0.21$, Serum P level $5.47 \pm 1.62 \mathrm{mg} / \mathrm{dl}$ and Serum PTH level $481.92 \pm 608.05 \mathrm{pg} / \mathrm{ml}$ as shown in Table 8. Also, the mean serum Triglyceride of the patients was $149.25 \pm 85.57 \mathrm{mg} / \mathrm{dl}$ and Cholesterol was $151.79 \pm 36.85$ (Table 2). 
SOHAG MEDICAL JOURNAL Study of hyperparathyroidism among patients with chronic

Table.2 Biochemical profile of all study population

\begin{tabular}{|c|c|}
\hline Variable & Summary statistics \\
\hline $\begin{array}{l}\text { Parathyroid hormone (PTH) } \\
\text { Mean } \pm \text { SD } \\
\text { Median (range) }\end{array}$ & $\begin{array}{c}481.92 \pm 608.05 \\
202.2(12.7-1900)\end{array}$ \\
\hline $\begin{array}{l}\text { Total calcium } \\
\text { Mean } \pm \text { SD } \\
\text { Median (range) } \\
\end{array}$ & $\begin{array}{c}8.69 \pm 1.30 \\
8.4(6.6-12.5)\end{array}$ \\
\hline $\begin{array}{l}\text { Ionized calcium } \\
\text { Mean } \pm \text { SD } \\
\text { Median (range) } \\
\end{array}$ & $\begin{array}{c}1.04 \pm 0.21 \\
1.00(0.7-1.85)\end{array}$ \\
\hline $\begin{array}{l}\text { Po4 } \\
\text { Mean } \pm \text { SD } \\
\text { Median (range) }\end{array}$ & $\begin{array}{c}5.47 \pm 1.62 \\
5.45(2.8-10.9)\end{array}$ \\
\hline $\begin{array}{l}\text { Triglyceride } \\
\text { Mean } \pm \text { SD } \\
\text { Median (range) } \\
\end{array}$ & $\begin{array}{c}149.25 \pm 85.57 \\
121.5(46-514)\end{array}$ \\
\hline $\begin{array}{l}\text { Cholesterol } \\
\text { Mean } \pm \text { SD } \\
\text { Median (range) }\end{array}$ & $\begin{array}{c}151.79 \pm 36.85 \\
142.5(80-295)\end{array}$ \\
\hline $\begin{array}{l}\text { LDL } \\
\text { Mean } \pm \text { SD } \\
\text { Median (range) }\end{array}$ & $\begin{array}{l}74.39 \pm 23.14 \\
70(34-155)\end{array}$ \\
\hline $\begin{array}{l}\text { HDL } \\
\text { Mean } \pm \text { SD } \\
\text { Median (range) }\end{array}$ & $\begin{array}{l}47.31 \pm 12.62 \\
44.5(21-89)\end{array}$ \\
\hline $\begin{array}{l}\text { VLDL } \\
\text { Mean } \pm \text { SD } \\
\text { Median (range) }\end{array}$ & $\begin{array}{c}28.98 \pm 15.85 \\
24(9-103)\end{array}$ \\
\hline
\end{tabular}

The echocardiographic manifestations were LVH was observed in $51(51.00 \%)$ patients, diastolic dysfunction (DD) as expressed by E/A ratio was present in $53(53.00 \%)$ patients, dilated left atrium was present in $19(19.00 \%)$ patients, dilated right side was present in $5(5.00 \%)$ patients. Mean LVEDD (left ventricular end diastolic diameter) was $4.90 \pm 0.97 \mathrm{~cm}$, mean EF (ejection fraction) was 59.92 \pm 6.93 (Table.3).

Other echocardiographic manifestations like IHD expressed as regular wall motion abnormality (hypokinesia) was observed in 17 (17.00\%) patients, pericardial Effusion was present in $14(14.00 \%)$ patients, and valvular calcification was present in $5(5.00 \%)$ patients (Table 4). 
SOHAG MEDICAL JOURNAL Study of hyperparathyroidism among patients with chronic

Table.3 Echocardiographic findings of all study population

\begin{tabular}{|c|c|}
\hline Variable & Summary statistics \\
\hline $\begin{array}{l}\text { LVH } \\
\text { No } \\
\text { Yes } \\
\end{array}$ & $\begin{array}{l}49(49.00 \%) \\
51(51.00 \%)\end{array}$ \\
\hline $\begin{array}{l}\text { EF } \\
\text { Mean } \pm \text { SD } \\
\text { Median (range) }\end{array}$ & $\begin{array}{c}59.92 \pm 6.93 \\
60(41-76) \\
\end{array}$ \\
\hline $\begin{array}{l}\text { E/A ratio } \\
\text { No diastolic dysfunction } \\
\text { Diastolic dysfunction }\end{array}$ & $\begin{array}{l}47(47.00 \%) \\
53(53.00 \%)\end{array}$ \\
\hline $\begin{array}{l}\text { Rt. Side } \\
\text { Normal } \\
\text { Dilated } \\
\end{array}$ & $\begin{array}{c}95(95.00 \%) \\
5(5.00 \%) \\
\end{array}$ \\
\hline $\begin{array}{l}\text { IVS } \\
\text { Mean } \pm \text { SD } \\
\text { Median (range) }\end{array}$ & $\begin{array}{c}1.69 \pm 0.46 \\
1.7(1.00-2.80)\end{array}$ \\
\hline $\begin{array}{l}\text { LVEDd } \\
\text { Mean } \pm \text { SD } \\
\text { Median (range) } \\
\end{array}$ & $\begin{array}{c}4.90 \pm 0.97 \\
4.8(2.75-6.93)\end{array}$ \\
\hline $\begin{array}{l}\text { LA diameter } \\
\text { Normal } \\
\text { Dilated }\end{array}$ & $\begin{array}{l}81(81.00 \%) \\
19(19.00 \%)\end{array}$ \\
\hline $\begin{array}{l}\text { M valve } \\
\text { Normal } \\
\text { Mild regurge } \\
\text { Rheumatic }\end{array}$ & $\begin{array}{c}64(64.00 \%) \\
32(32.00 \%) \\
4(4.00 \%) \\
\end{array}$ \\
\hline $\begin{array}{l}\text { T valve } \\
\text { Normal } \\
\text { Mild regurge } \\
\text { Rheumatic } \\
\end{array}$ & $\begin{array}{c}75(75.00 \%) \\
23(23.00 \%) \\
2(2.00 \%) \\
\end{array}$ \\
\hline $\begin{array}{l}\text { A valve } \\
\text { Normal } \\
\text { Mild regurge } \\
\text { Rheumatic } \\
\end{array}$ & $\begin{array}{c}91(91.00 \%) \\
4(4.00 \%) \\
5(5.00 \%) \\
\end{array}$ \\
\hline
\end{tabular}


SOHAG MEDICAL JOURNAL Study of hyperparathyroidism among patients with chronic

Table.4 Echocardiographic findings of all study population, continued

\begin{tabular}{|c|c|}
\hline Variable & Summary statistics \\
\hline $\begin{array}{l}\text { PHTN } \\
\text { No } \\
\text { Yes }\end{array}$ & $\begin{array}{c}93(93.00 \%) \\
7(93.00 \%)\end{array}$ \\
\hline $\begin{array}{l}\text { Valve calcification } \\
\text { No } \\
\text { Yes }\end{array}$ & $\begin{array}{c}95(95.00 \%) \\
5(5.00 \%) \\
\end{array}$ \\
\hline $\begin{array}{l}\text { Pericardial disease } \\
\text { No } \\
\text { Yes }\end{array}$ & $\begin{array}{l}86(86.00 \%) \\
14(14.00 \%)\end{array}$ \\
\hline $\begin{array}{l}\text { Regular wall motion } \\
\text { Normal } \\
\text { Hypokinesia }\end{array}$ & $\begin{array}{l}83(83.00 \%) \\
17(17.00 \%)\end{array}$ \\
\hline $\begin{array}{l}\text { RHD } \\
\text { No } \\
\text { Yes }\end{array}$ & $\begin{array}{c}94(94.00 \%) \\
6(6.00 \%)\end{array}$ \\
\hline
\end{tabular}

Table.5 Prevalence of hyperparathyroidism of all study population

\begin{tabular}{|l||c|}
\hline Variable & Summary statistics \\
\hline \hline Hyperparathyroidism & $34(34.00 \%)$ \\
No & $66(66.00 \%)$ \\
Yes & \\
\hline
\end{tabular}

Table 5 and Figure 1 show that the prevalence of hyperparathyroidism was (66.00\%) in the study.

Figure.1 Prevalence of hyperparathyroidism of all study population

\section{Hyperparathyroidism}

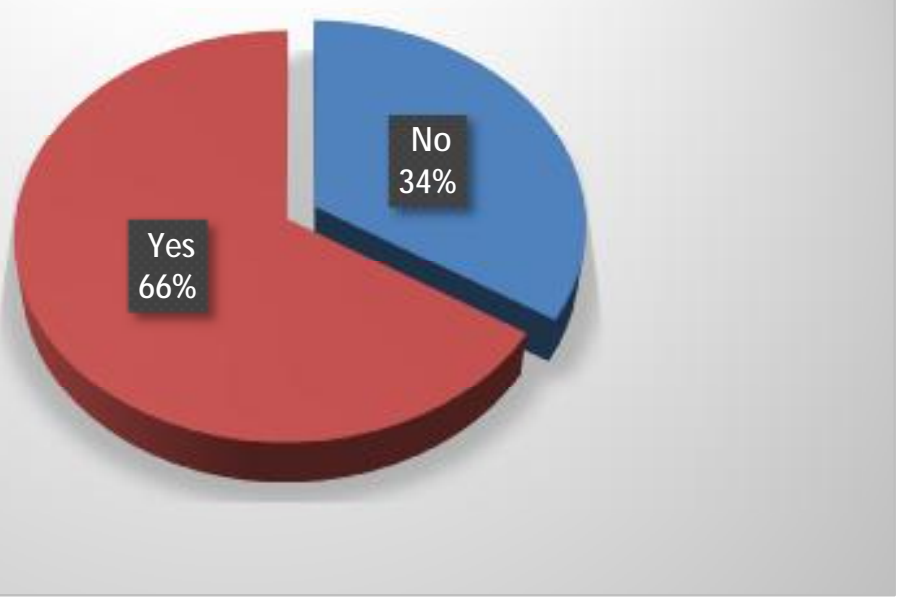


SOHAG MEDICAL JOURNAL Study of hyperparathyroidism among patients with chronic Vol. 21 No.3 october 2017 Mahmoud Kh. Mahmoud

Table.6 Relation between CKD stage and bone density of study populations

\begin{tabular}{|c|c|c|c|c|c|c|c|}
\hline Variable & $\begin{array}{c}\text { Stage (1- } \\
2)\end{array}$ & $\begin{array}{c}\text { Stage (3- } \\
\text { 4) }\end{array}$ & Stage (5) & $\mathbf{P}$ & P1 & P2 & P3 \\
\hline $\begin{array}{l}\text { Bone } \\
\text { density } \\
\text { Normal } \\
\text { Osteopenia } \\
\text { Osteoporos } \\
\text { is } \\
\text { Osteomala } \\
\text { cia }\end{array}$ & $\begin{array}{c}13 \\
(65.00 \%) \\
6 \\
(30.00 \%) \\
1 \\
(5.00 \%) \\
0\end{array}$ & $\begin{array}{c}12 \\
(60.00 \%) \\
4 \\
(20.00 \%) \\
3 \\
(15.00 \%) \\
1 \\
(5.00 \%)\end{array}$ & $\begin{array}{c}30(50.00 \%) \\
19(31.67 \%) \\
9(15.00 \%) \\
2(3.33 \%)\end{array}$ & 0.73 & 0.49 & 0.47 & 0.78 \\
\hline
\end{tabular}

$P$ compared the 3 groups, P1 compare stage (1-2) to stage (3-4), P2 compare stage

(1-2) to stage (5), and P3 compare stage (3-4) to stage (5)

The patients were divided into three groups according to CKD stage; stage (1-2) that included $20(20.00 \%)$ patients, stage (3-4) that included $20(20.00 \%)$ patients, and stage

(5) that included $60(60.00 \%)$ patients

Table.7 Relation between CKD stage and biochemical profile of study populations

\begin{tabular}{|c|c|c|c|c|c|c|c|}
\hline Variable & Stage (1-2) & Stage (3-4) & Stage (5) & $\mathbf{P}$ & P1 & $\mathbf{P 2}$ & P3 \\
\hline $\begin{array}{l}\text { PTH } \\
\text { Mean } \pm \text { SD } \\
\text { Median (range) }\end{array}$ & $\begin{array}{c}66.54 \pm 45.80 \\
56.55(12.7- \\
210.8)\end{array}$ & $\begin{array}{c}142.04 \pm 45.8 \\
91.45(20.4- \\
393.9)\end{array}$ & $\begin{array}{c}733.7 \pm 673.1 \\
438.9(27.3- \\
1900)\end{array}$ & 0.0001 & 0.10 & 0.0001 & 0.0001 \\
\hline $\begin{array}{l}\text { Total calcium } \\
\text { Mean } \pm \text { SD } \\
\text { Median (range) }\end{array}$ & $\begin{array}{c}9.44 \pm 1.02 \\
9.5(7.9-11.8)\end{array}$ & $\begin{array}{c}8.32 \pm 0.98 \\
8.05(6.8-10.2)\end{array}$ & $\begin{array}{c}8.57 \pm 1.39 \\
8.1(6.6-12.5)\end{array}$ & 0.01 & 0.02 & 0.02 & 1.00 \\
\hline $\begin{array}{l}\text { Ionized calcium } \\
\text { Mean } \pm \text { SD } \\
\text { Median (range) }\end{array}$ & $\begin{array}{c}1.16 \pm 0.15 \\
1.2(0.9-1.5)\end{array}$ & $\begin{array}{c}0.98 \pm 0.17 \\
0.91(0.8-1.82)\end{array}$ & $\begin{array}{c}1.02 \pm 0.22 \\
1.0(0.7-1.85)\end{array}$ & 0.001 & 0.001 & 0.0009 & 0.63 \\
\hline $\begin{array}{l}\text { Po4 } \\
\text { Mean } \pm \text { SD } \\
\text { Median (range) }\end{array}$ & $\begin{array}{c}4.16 \pm 1.00 \\
4.1(2.8-6.1)\end{array}$ & $\begin{array}{c}5.49 \pm 1.15 \\
5.75(3.1-7.8)\end{array}$ & $\begin{array}{c}5.91 \pm 1.68 \\
5.9(3.0-10.9)\end{array}$ & 0.0001 & 0.01 & $<0.0001$ & 0.85 \\
\hline $\begin{array}{l}\text { Triglyceride } \\
\text { Mean } \pm \text { SD } \\
\text { Median (range) }\end{array}$ & $\begin{array}{l}130.9 \pm 59.02 \\
121(60-274)\end{array}$ & $\begin{array}{c}142.4 \pm 94.39 \\
117.5(46-446)\end{array}$ & $\begin{array}{c}157.7 \pm 89.95 \\
125.5(51-514)\end{array}$ & 0.51 & 0.88 & 0.31 & 0.92 \\
\hline $\begin{array}{l}\text { Cholesterol } \\
\text { Mean } \pm \text { SD } \\
\text { Median (range) }\end{array}$ & $\begin{array}{c}159.5 \pm 50.34 \\
144.5(95-270)\end{array}$ & $\begin{array}{c}147.5 \pm 31.37 \\
138(117-247)\end{array}$ & $\begin{array}{r}150.7 \pm 33.43 \\
145(80-295)\end{array}$ & 0.87 & 0.92 & 0.92 & 0.52 \\
\hline $\begin{array}{l}\text { LDL } \\
\text { Mean } \pm \text { SD } \\
\text { Median (range) }\end{array}$ & $\begin{array}{c}74.1 \pm 20.68 \\
63.5(50-110) \\
\end{array}$ & $\begin{array}{l}68.85 \pm 20.58 \\
63(40-117) \\
\end{array}$ & $\begin{array}{l}76.85 \pm 24.70 \\
70(34-155) \\
\end{array}$ & 0.45 & 0.42 & 0.65 & 0.22 \\
\hline $\begin{array}{l}\text { HDL } \\
\text { Mean } \pm \text { SD } \\
\text { Median (range) }\end{array}$ & $\begin{array}{r}53.2 \pm 17.77 \\
47(34-89) \\
\end{array}$ & $\begin{array}{r}46.7 \pm 11.73 \\
42.5(32-66)\end{array}$ & $\begin{array}{c}45.5 \pm 10.30 \\
44(21-71) \\
\end{array}$ & 0.06 & 0.30 & 0.06 & 1.00 \\
\hline $\begin{array}{l}\text { VLDL } \\
\text { Mean } \pm \text { SD } \\
\text { Median (range) }\end{array}$ & $\begin{array}{c}25.7 \pm 11.80 \\
23.5(12-55) \\
\end{array}$ & $\begin{array}{c}28.45 \pm 18.81 \\
23.5(9-89) \\
\end{array}$ & $\begin{array}{l}30.25 \pm 16.04 \\
24(12-103)\end{array}$ & 0.53 & 0.97 & 0.30 & 0.46 \\
\hline
\end{tabular}


SOHAG MEDICAL JOURNAL Study of hyperparathyroidism among patients with chronic

P compared the 3 groups, P1 compare stage (1-2) to stage (3-4),P2 compare stage (12) to stage (5), and P3 compare stage (3-4) to stage (5)

There was significant positive correlation between three groups of study population and serum PTH levels $\mathrm{p}$ value $=0.0001$ and correlation between each two groups and serum PTH levels $\mathrm{p}_{1}=0.1, \mathrm{p}_{2}=0.0001, \mathrm{p}_{3}=0.0001$ (Table 7).

Also, there was positive significant correlation between three groups and serum $\mathrm{Po}_{4} \mathrm{p}$ value $=0.0001$ and correlation between each two groups and serum $\mathrm{Po}_{4}$ levels $\mathrm{p}_{1}=0.01$, $\mathrm{p}_{2}<0.0001$, but no significant correlation between severe CKD and stage $5 \mathrm{p}_{3}=0.85$ (Table 7).

While, there was negative significant correlation between three groups and serum total and ionized calcium $\mathrm{p}$ value $=0.01, \mathrm{p}$ value $=0.001$ respectively and correlation between each two groups and serum total and ionized calcium levels $\mathrm{p}_{1}=0.02 \mathrm{p}_{1}=0.001$ respectively, $\mathrm{p}_{2}=0.02 \mathrm{p}_{2}=0.0009$ respectively, but also no significant correlation between severe CKD (stage 3-4) and stage $5 \mathrm{p}_{3}=1.00 \mathrm{p}_{3}=0.63$ respectively (Table 7).

Table.8 Relation between CKD stage and ECG findings of study populations

\begin{tabular}{|c|c|c|c|c|c|c|c|}
\hline Variable & Stage (1-2) & Stage (3-4) & Stage (5) & $\mathbf{P}$ & P1 & $\mathbf{P 2}$ & P3 \\
\hline $\begin{array}{l}\text { LVH } \\
\text { No } \\
\text { Yes }\end{array}$ & $\begin{array}{c}19 \\
(95.00 \%) \\
1(5.00 \%)\end{array}$ & $\begin{array}{c}12 \\
(60.00 \%) \\
8(40.00 \%)\end{array}$ & $\begin{array}{c}35 \\
(58.33 \%) \\
25 \\
(41.67 \%)\end{array}$ & 0.009 & 0.02 & 0.002 & 1.00 \\
\hline $\begin{array}{l}\text { RVH } \\
\text { No } \\
\text { Yes }\end{array}$ & $\begin{array}{c}18 \\
(90.00 \%) \\
2(10.00 \%) \\
\end{array}$ & $\begin{array}{c}20(100 \%) \\
0\end{array}$ & $\begin{array}{c}58 \\
(96.67 \%) \\
2(3.33) \\
\end{array}$ & 0.25 & 0.45 & 0.26 & 1.00 \\
\hline $\begin{array}{l}\text { P wave morphology } \\
\text { Normal } \\
\text { P mitral }\end{array}$ & $\begin{array}{c}20(100 \%) \\
0\end{array}$ & $\begin{array}{c}20(100 \%) \\
0\end{array}$ & $\begin{array}{c}53 \\
(88.33 \%) \\
7(11.67 \%) \\
\end{array}$ & 0.08 & 1.0 & 0.18 & 0.18 \\
\hline $\begin{array}{l}\text { Ischemia } \\
\text { No } \\
\text { Yes }\end{array}$ & $\begin{array}{c}16 \\
(80.00 \%) \\
4(20.00 \%)\end{array}$ & $\begin{array}{c}19 \\
(95.00 \%) \\
1(5.00 \%)\end{array}$ & $\begin{array}{c}48 \\
(80.00 \%) \\
12 \\
(20.00 \%) \\
\end{array}$ & 0.27 & 0.34 & 1.00 & 0.17 \\
\hline $\begin{array}{l}\text { Rate } \\
\text { Normal } \\
\text { Sinus tachycardia }\end{array}$ & $\begin{array}{c}19 \\
(95.00 \%) \\
1(5.00 \%) \\
\end{array}$ & $\begin{array}{c}17 \\
(75.00 \%) \\
3(15.00 \%)\end{array}$ & $\begin{array}{c}56 \\
(93.33 \%) \\
4(6.67 \%) \\
\end{array}$ & 0.42 & 0.61 & 1.00 & 0.36 \\
\hline $\begin{array}{l}\text { Arrhythmia } \\
\text { Normal } \\
\text { AF } \\
\text { PVC }\end{array}$ & $\begin{array}{c}17 \\
(\mathbf{8 5 . 0 0 \% )} \\
\mathbf{3}(\mathbf{1 5 . 0 0 \% )}) \\
1(\mathbf{5 . 0 0 \% )}\end{array}$ & $\begin{array}{c}20(100 \%) \\
0 \\
0\end{array}$ & $\begin{array}{c}54 \\
(90.00 \%) \\
2(3.33 \%) \\
4(6.67 \%)\end{array}$ & 0.08 & 0.23 & 0.10 & 0.75 \\
\hline
\end{tabular}

P compared the 3 groups, P1 compare stage (1-2) to stage (3-4),P2 compare stage (1-

2) to stage (5), and P3 compare stage (3-4) to stage (5)

When we compared three groups of CKD patients to ECG findings, we found significant correlation between CKD stages and LVH $p$ value $=0.09$ and correlation between each two groups and $\mathrm{LVH} \mathrm{p}_{1}$ value $=0.02$ and $\mathrm{p}_{2}$ value $=0.002$, while no significant correlation between severe CKD and stage $5 \mathrm{p}_{3}$ value $=1.00$. 
SOHAG MEDICAL JOURNAL Study of hyperparathyroidism among patients with chronic

\section{Table.9 Relation between CKD stage and echocardiographic findings of study} populations

\begin{tabular}{|c|c|c|c|c|c|c|c|}
\hline Variable & Stage (1-2) & Stage (3-4) & Stage (5) & $\mathbf{P}$ & P1 & $\mathbf{P 2}$ & P3 \\
\hline $\begin{array}{l}\text { LVH } \\
\text { No } \\
\text { Yes } \\
\end{array}$ & $\begin{array}{c}17(85.0 \%) \\
3(15.0 \%)\end{array}$ & $\begin{array}{c}12(60.0 \%) \\
8(40.0 \%)\end{array}$ & $\begin{array}{l}20(33.33 \%) \\
40(66.67 \%)\end{array}$ & $<0.0001$ & 0.08 & $<0.0001$ & 0.04 \\
\hline $\begin{array}{l}\mathbf{E F} \\
\text { Mean } \pm \text { SD } \\
\text { Median (range) } \\
\end{array}$ & $\begin{array}{c}63.6 \pm 4.04 \\
63.5(53-70) \\
\end{array}$ & $\begin{array}{c}60 \pm 6.31 \\
57.5(52-74) \\
\end{array}$ & $\begin{array}{r}58.71 \pm 7.54 \\
57.5(41-76)\end{array}$ & 0.03 & 0.32 & 0.02 & 1.00 \\
\hline $\begin{array}{l}\text { E/A ratio } \\
\text { No diastolic dysfunction } \\
\text { Diastolic dysfunction }\end{array}$ & $\begin{array}{c}19(95.0 \%) \\
5(5.0 \%)\end{array}$ & $\begin{array}{c}11(55.0 \%) \\
9(45.0 \%)\end{array}$ & $\begin{array}{l}17(28.33 \%) \\
43(71.67 \%)\end{array}$ & $<0.0001$ & 0.003 & $<0.0001$ & 0.03 \\
\hline $\begin{array}{l}\text { Rt. Side } \\
\text { Normal } \\
\text { Dilated }\end{array}$ & $\begin{array}{l}19(95.00) \\
1(5.00 \%)\end{array}$ & $\begin{array}{c}19(95.0 \%) \\
1(5.00 \%)\end{array}$ & $\begin{array}{c}57(95.0 \%) \\
3(5.0 \%)\end{array}$ & 1.00 & 1.00 & 1.00 & 1.00 \\
\hline $\begin{array}{l}\text { IVS } \\
\text { Mean } \pm \text { SD } \\
\text { Median (range) }\end{array}$ & $\begin{array}{c}1.56 \pm 0.56 \\
1.4(1.09- \\
2.8)\end{array}$ & $\begin{array}{c}1.68 \pm 0.54 \\
1.7(1.0-2.5)\end{array}$ & $\begin{array}{c}1.73 \pm 0.39 \\
1.8(1.0-2.4)\end{array}$ & 0.21 & 0.56 & 0.06 & 0.71 \\
\hline $\begin{array}{l}\text { LVEDd } \\
\text { Mean } \pm \text { SD } \\
\text { Median (range) }\end{array}$ & $\begin{array}{l}4.49 \pm 0.86 \\
4.47(3.02- \\
6.1)\end{array}$ & $\begin{array}{l}4.61 \pm 0.80 \\
4.7(3.02- \\
5.88)\end{array}$ & $\begin{array}{l}5.13 \pm 0.99 \\
5.2(2.75- \\
6.93)\end{array}$ & 0.01 & 1.00 & 0.02 & 0.10 \\
\hline $\begin{array}{l}\text { LA diameter } \\
\text { Normal } \\
\text { Dilated }\end{array}$ & $\begin{array}{l}18(80.0 \%) \\
2(10.00 \%)\end{array}$ & $\begin{array}{l}17(85.0 \%) \\
3(15.00 \%)\end{array}$ & $\begin{array}{l}46(76.67 \%) \\
14(23.33 \%)\end{array}$ & 0.37 & 1.00 & 0.33 & 0.54 \\
\hline $\begin{array}{l}\text { M valve } \\
\text { Normal } \\
\text { Mild regurge } \\
\text { Rheumatic }\end{array}$ & $\begin{array}{l}16(80.0 \%) \\
2(10.0 \%) \\
2(10.0 \%)\end{array}$ & $\begin{array}{c}15(75.0 \%) \\
5(25.0 \%) \\
0\end{array}$ & $\begin{array}{c}33(55.0 \%) \\
25(41.67 \%) \\
2(3.33 \%)\end{array}$ & 0.046 & 0.19 & 0.03 & 0.45 \\
\hline $\begin{array}{l}\text { T valve } \\
\text { Normal } \\
\text { Mild regurge } \\
\text { Rheumatic }\end{array}$ & $\begin{array}{c}20(100 \%) \\
0 \\
0 \\
\end{array}$ & $\begin{array}{c}19(95.0 \%) \\
1(5.0 \% \\
0\end{array}$ & $\begin{array}{c}36(60.00 \%) \\
22(36.67 \%) \\
2(3.33 \%) \\
\end{array}$ & 0.001 & 0.31 & 0.003 & 0.01 \\
\hline $\begin{array}{l}\text { A valve } \\
\text { Normal } \\
\text { Mild regurge } \\
\text { Rheumatic }\end{array}$ & $\begin{array}{c}17(85.0 \%) \\
1(5.0 \%) \\
2(10.0 \%)\end{array}$ & $\begin{array}{c}20(100 \%) \\
0 \\
0\end{array}$ & $\begin{array}{c}54(90.0 \%) \\
3(5.00 \%) \\
3(5.00 \%)\end{array}$ & 0.51 & 0.20 & 0.73 & 0.34 \\
\hline
\end{tabular}

P compared the 3 groups, P1 compare stage (1-2) to stage (3-4),P2 compare stage (1-

2) to stage (5), and P3 compare stage (3-4) to stage (5)

When we compared three groups of CKD patients to Echocardiographic findings, we found significant correlation between CKD stages and LVH $p$ value $<0.0001$ and correlation between each two groups and LVH $\mathrm{p}_{1}$ value $=0.08$ and $\mathrm{p}_{2}$ value $<0.0001$, and $\mathrm{p}_{3}$ value $=0.04$ (Table 9$)$.

Our results found correlation between stages CKD patients and EF with $p$ value $=0.03$

(Table 9), and LVEDd with p value $=0.01$ (Table 16).

Also, there was significant correlation between CKD stages in diastolic dysfunction (DD) that was denoted by E/A ratio $\mathrm{p}$ value $<0.0001, \quad \mathrm{p}_{1}$ value $=0.003, \mathrm{p}_{2}$ value $<$ 0.0001 , and $\mathrm{p}_{3}$ value $=0.03($ Table16) 
SOHAG MEDICAL JOURNAL Study of hyperparathyroidism among patients with chronic Vol. 21 No.3 october 2017 Mahmoud Kh. Mahmoud

Table.10 Relation between CKD stage and echocardiographic findings of study populations, continued

\begin{tabular}{|c|c|c|c|c|c|c|c|}
\hline Variable & Stage (1-2) & Stage (3-4) & Stage (5) & $\mathbf{P}$ & P1 & $\mathbf{P 2}$ & P3 \\
\hline $\begin{array}{l}\text { PHTN } \\
\text { No } \\
\text { Yes }\end{array}$ & $\begin{array}{c}20(100 \%) \\
0\end{array}$ & $\begin{array}{c}19 \\
(95.0 \%) \\
1(5.0 \%)\end{array}$ & $\begin{array}{c}54 \\
(90.00 \%) \\
6(10.33 \%)\end{array}$ & 0.29 & 1.00 & 0.33 & 0.67 \\
\hline $\begin{array}{l}\text { Valve calcification } \\
\text { No } \\
\text { Yes }\end{array}$ & $\begin{array}{c}20(100 \%) \\
0\end{array}$ & $\begin{array}{c}20(100 \%) \\
0\end{array}$ & $\begin{array}{c}55 \\
(91.67 \%) \\
5(8.33 \%) \\
\end{array}$ & 0.17 & 1.0 & 0.32 & 0.32 \\
\hline $\begin{array}{l}\text { Pericardial disease } \\
\text { No } \\
\text { Yes }\end{array}$ & $\begin{array}{c}19 \\
(95.0 \%) \\
1(5.00 \%)\end{array}$ & $\begin{array}{c}17 \\
(85.0 \%) \\
3 \\
(15.00 \%)\end{array}$ & $\begin{array}{c}50 \\
(83.33 \%) \\
10 \\
(16.67 \%)\end{array}$ & 0.42 & 0.61 & 0.28 & 1.00 \\
\hline $\begin{array}{l}\text { Regular wall motion } \\
\text { Normal } \\
\text { Hypokinesia }\end{array}$ & $\begin{array}{c}20(100 \%) \\
0\end{array}$ & $\begin{array}{c}20(100 \%) \\
0\end{array}$ & $\begin{array}{c}43 \\
(71.67 \%) \\
17 \\
(28.33 \%)\end{array}$ & 0.001 & 1.00 & 0.005 & 0.005 \\
\hline $\begin{array}{l}\text { RHD } \\
\text { No } \\
\text { Yes }\end{array}$ & $\begin{array}{c}18 \\
(90.0 \%) \\
2(10.0 \%)\end{array}$ & $\begin{array}{c}20(100 \%) \\
0\end{array}$ & $\begin{array}{c}56 \\
(93.33 \%) \\
4(6.67 \%)\end{array}$ & 0.39 & 0.49 & 0.64 & 0.57 \\
\hline
\end{tabular}

$P$ compared the 3 groups, P1 compare stage (1-2) to stage (3-4),P2 compare stage (12) to stage (5D), and P3 compare stage (3-4) to stage (5)

Table 10 showed significant correlation between different stages of CKD patients and RWMA (hypokinesia) i.e. ischemia with $\mathrm{p}$ value $=0.001$, but no significant correlation between different stages of CKD predialysis patients $\mathrm{p}_{1}$ value $=1.00$, while there was significant correlation between each stage CKD predialysis patients (stage 1-2, and stage $3-4)$ and CKD (stage 5) dialysis patients $\mathrm{p}_{2}$ value $=0.005$, and $\mathrm{p}_{3}$ value $=0.005 \quad$ (Table 10).

\section{DISCUSSION}

Secondary hyperparathyroidism is a common complication of chronic kidney disease (CKD), and is characterized by elevated levels of serum parathyroid hormone (PTH) and abnormalities in bone and mineral metabolism. ${ }^{4}$

This serious disorder arises from disturbances in the regulation of the intracellular and extracellular levels of PTH, calcium, phosphorus, and vitamin D (calcitriol), which become more severe as kidney function declines and the interaction between these factors is complex, and effective control of secondary HPT can create a significant challenge. ${ }^{15}$
This study was conducted on 100 patients with different stages of chronic kidney disease in Sohag University Hospital. The reference range for serum PTH was $12-72 \mathrm{pg} / \mathrm{ml}$.

66 patients have PTH above $72 \mathrm{pg} / \mathrm{ml}$, this means that the prevalence of secondary hyperparathyroidism in our study is $66.00 \%$, with mean $\mathrm{PTH}=$ $481.92 \pm 608.05$. This prevalence is in agreement with MalawadiBn et al in 2014, who studied the prevalence of secondary hyperparathyroidism in 150 patients with chronic kidney disease different stages.Although this prevalence lower than that that was estimated by 
SOHAG MEDICAL JOURNAL Study of hyperparathyroidism among patients with chronic

Owda et al in 2003, who studied the prevalence of secondary hyperparathyroidism in 122 patients with chronic kidney disease and estimated a prevalence of $78 \%$ with mean $\mathrm{iPTH}=$ $843 \pm 184$ (Owda et aL, 2003), but this difference can be explained by the difference in the stages of CKD patients, that were only stage 5 by Owda et aL, 2003. Although earlier study done by Salem in 1997 estimated a prevalence of $50 \%$ which is much lower than that we and Owda et al 2003, have estimated, but this difference can be explained by the difference in the time on dialysis, that was underestimated by Salem in 1997 when he included all patients on hemodialysis.

The level of serum PTH was higher in more advanced renal failure thus confirming the relationship between severity of hyperparathyroidism and the degree of renal impairment. ${ }^{16}$ Our results are also in agreement with results reported by Malawadi et al., who demonstrated that The serum PTH $(331.68 \pm 204.99 \mathrm{pg} / \mathrm{ml})$ was significantly higher in more advanced renal failure(CKD stage 5) which confirms the relationship between severity of hyperparathyroidism and the degree of renal impairment therefore, serum PTH is negatively correlated with creatinine clearance $(p<0.001)$.

In this study, Serum phosphorus was found to be significantly positively correlated to PTH both in the total study population ( $\mathrm{p}$-value $=0.0001)$ and in patients with hyperparathyroidism (pvalue $=0.006$ ). Our results are in agreement with results reported by Gamal et al., 2005.

In the present study the total serum calcium goes on decreasing as the stage of CKD advances. The PTH has a significant negative correlation with serum total calcium both in the total study population $(\mathrm{p}$-value $=0.01)$ and in patients with hyperparathyroidism ( $\mathrm{p}$ value $=0.03$ ) and these results keeping with that estimated by Malawadi et al in 2014, serum PTH is negatively correlated with serum total calcium $(\mathrm{p}<0.001)$.

We found that the time on dialysis is significantly correlated with PTH levels where, there is linear correlation between the duration of hemodialysis and PTH levels p-value $=0.0001$. Our results keeping with that estimated by Chertow et al in 2000 and Owda et al in 2003, although study by Gamal et al et al in 2005 found that the time of dialysis not significantly correlated with PTH levels. This difference may be explained in view of our study included patients who have been on dialysis for at least 1 year, but Gamal et al study included patients who have been on dialysis for much shorter durations.

Four main structural abnormalities of the heart have been described in patients with CKD: LV hypertrophy, expansion of the nonvascular cardiac interstitium leading to intermyocardiocytic fibrosis, changes in vascular architecture, and myocardial calcification. ${ }^{133}$

Left ventricular hypertrophy was reported in $51(51.00 \%)$ patients. It is keeping with that reported by Menon et al in 1998 who reported $40 \%$ incidence. But not consistent with Raut et al in 1998, who reported $30 \%$ incidence, and Goornavar et al in $\mathbf{2 0 0 5}^{138}$ who reported $36 \%$ incidence, and this is explained by decreased number of stages of CKD predialysis patients in their studies (8\% in Goornavar et al), but our study included $40 \%$ of all study populations, and In the present study statistically significant correlation was observed between left ventricular 
SOHAG MEDICAL JOURNAL Study of hyperparathyroidism among patients with chronic

hypertrophy and stage of the chronic kidney disease ( $p$-value $<0.0001$ ) and duration of dialysis $(\mathrm{p}$-value $=0.001)$.

In the present study ischemic heart disease was documented in $17(17.00 \%)$ patients echo, in consistent with Goornovar et al who reported 16\% incidence of ischemic heart disease, but in contrast to Parfrey et al

Pericardial effusion was reported in 14 $(14.00 \%)$ patients which is consistent with a study by Laddha M et al 2014 where they found $14.3 \%$ patients with pericardial effusion, and Shvendra et al who reported an incidence of $17.14 \%$, but is not consistent with Goornvar et al who reported incidence of $6 \%$ patients and Barrionuevo JDA et al (2010) et al where they found $6.5 \%$ patients with pericardial effusion, While Menon et al (1998) who reported $32 \%$ incidence and Achari et al (1989) who reported 50\% incidence of pericardial effusion in chronic kidney disease patients, this is due to this study included CKD patients with different stages but most of other studies included CKD patients on dialysis, also hypervolemia and insufficient dialysis are co-factors affect in the results.

Diastolic dysfunction as denoted by E/A ratio was present in $53(53.00 \%)$ of patients, which is consistent with Laddha $M$ et al 2014 who reported an incidence $61.4 \%$ of patients with diastolic dysfunction.

Valvular calcification was noted in 5\% of CKD patients, which is consistent with Laddha M et al 2014 who reported an incidence $7.1 \%$ of patients.

\section{Conclusion}

We can conclude that the prevalence of secondary hyperparathyroidism in chronic kidney disease is high In our study (as regard cardiovascular risk) as regard echocardiography in CKD patients; maladaptive events leading to $\mathrm{LVH}$, structural changes myocardium as well as diastolic dysfunction and even systolic failure occur frequently.

\section{References}

1. Meguid El Nahas A, Bello AK. Chronic kidney disease: the global challenge. Lancet 2005; 365:331-40.

2. Go AS, Chertow GM, Fan D, McCulloch CE, Hsu CY. Chronic kidney disease and the risks of death, cardiovascular events, and hospitalization N Engl J Med 2004; 351:1296-305.

3. Covic A, Kothawala P, Bernal M, Robbins S, Chalian A, Goldsmith D. Systematic review of the evidence underlying the association between mineral metabolism disturbances and risk of all-cause mortality, cardiovascular mortality and cardiovascular events in chronic kidney disease. Nephrol Dial Transplant 2009; 24:1506-23.

4. Slatopolsky E, Delmez JA. Pathogenesis of secondary hyperparathyroidism. Am J Kidney Dis 2009; 23:229-236

5. Slatopolsky E, Finch J, Denda M, Ritter C, Zhong M, Dusso , MacDonald PN, Brown AJ: Phosphorus restriction prevents parathyroid gland growth. High phosphorus directly stimulates PTH secretion in vitro. J Clin Invest 2003; 97 : 2534-2540.

6. Drüeke TB. Cell Biology of Parathyroid Gland Hyperplasia in Chronic Renal Failure. AmSocNephrol 11:1141-1152, 2010.

7. Brown EM: Gamba G, Ricardi D, et al. Cloning and characterization of an extracellular ca(2+)-sensing receptor from bovine parathyroid. Nature 1993; 366: 575-80.

8. Bikle DD: Clinical counter point. Vitamin D: New actions, new 
SOHAG MEDICAL JOURNAL Study of hyperparathyroidism among patients with chronic

analogs,new therapeutic potential. Endocr Rev13: 765-784, 2009.

9.Yalcindag C, Silver J, Naveh-Many T: Mechanism of increased parathyroid hormone mRNA in experimental uremia: Roles of protein RNA and RNA degradation. J Am Soc Nephrol10: 25622568, 1999.

10.Slatopolsky E, Delmez JA. Pathogenesis of secondary hyperparathyroidism. Am J Kidney Dis 2009; 23:229-236

11.Moe S, Drueke T, Cunningham J, et al. Definition, evaluation, and Classification of renal osteodystrophy: a position statement from Kidney Disease: Improving Global Outcomes (KDIGO). Kidney Int 2006; 69:1945-53.

12.Schafer C, Heiss A, Schwarz A, et al. The serum protein alpha 2- HeremansSchmid glycoprotein/fetuin-A is a systemically acting inhibitor of ectopic calcification. J Clin Invest 2003; 112:357-66.
13.Cannata-Andia JB, Rodriguez-Garcia $M$, Carrillo-Lopez N, Naves-Diaz M, DiazLopez B. Vascular calcifications: pathogenesis, management, and impact on clinical outcomes. J Am SocNephrol 2006; 17:S267-73.

14.Massy ZA, Drueke TB. Vascular calcification.

CurrOpinNephrolHypertens 2013; 22:405-12.

15. Shanahan CM, Cary NR, Metcalfe JC, Weissberg PL: High expression of genes for calcification-regulating proteins in human atherosclerotic plaques. J Clin Invest 2003; 93: 2393-2402.

16.Ian H, IninaGorodetskaya, Belinda Young, Chi-yuan, Glenn MC. The severity of secondary hyperpatathyeoidism in chronic renal insufficiency is GFR dependent, race dependent and associated with cardiovascular disease. 